\title{
An integrated hot-stage microscope-Direct Analysis in Real-Time-mass spectrometry system for studying the thermal behavior of materials
}

\author{
Gage P. Ashton, Lindsay P. Harding* and Gareth M.B. Parkes*
}

Dept of Chemical Sciences, University of Huddersfield, Queensgate, Huddersfield, HD1 3DH, UK.

\begin{abstract}
This paper describes a new analytical instrument that combines a precisely temperature-controlled hot-stage with digital microscopy and Direct Analysis in Real-Time mass spectrometry (DART-MS) detection. The novelty of the instrument lies in its ability to monitor processes as a function of temperature through the simultaneous recording of images, quantitative color changes and mass spectra. The capability of the instrument was demonstrated through successful application to four very varied systems including profiling an organic reaction, decomposition of silicone polymers and the desorption of rhodamine B from an alumina surface. The multi-dimensional, real-time analytical data provided by this instrument allow for a much greater insight into thermal processes than could be achieved previously.
\end{abstract}

Hot-stage microscopy (also called thermomicroscopy) is a well-established thermal analysis technique for studying the optical properties of a material as a function of temperature. ${ }^{1}$ Hot-stages coupled with compound microscopes, often with polarising filters, have been used to study the melting, nucleation and crystal growth processes in organic materials such as pharmaceuticals. ${ }^{2}$ Hot-stages coupled with stereoscopic microscopes allow for the study of opaque materials, either as solids or powders, and thermal processes ranging from melting to decomposition can be monitored. Most systems allow for the recording of images either as videos or a series of pictures taken at set time or temperature intervals. Others can provide additional quantitative information from recording the intensity of light reflected from the surface of the sample ${ }^{3}$ or obtaining information relating to color changes based on real-time pro-cessing of digital images. ${ }^{4}$

Direct Analysis in Real-Time is an ionisation technique developed in 2005 which is used with mass spectrometry detection; the combined technique is known as DARTMS. ${ }^{5}$ DART is a so-called ambient ionisation technique whereby solids, liquids and solutions can be analysed directly without the need for sample preparation which has opened up many new application areas in forensics 6 , food $^{7}$ and quality control. ${ }^{8}$

Samples are placed into the DART source under ambient conditions and a stream of heated gas containing metastable helium ions is directed at the sample surface. This process desorbs and ionises analyte molecules which then enter the mass spectrometer for analysis.

Since its inception, many hundreds of papers have been published using DART-MS. A good overview of the fundamentals and scope of the technique has been given by Gross $^{9}$ which gives some of the many systems to which DART-MS has been successfully applied. 
The vast majority of papers report applications of the technique and there are very few investigations into the fundamental effects of changing experimental parameters such as temperature, gas flow rate, sample introduction method etc. Recently, we have published results which showed that sample positioning, gas flow rate and the construction material and geometry of the sample holder greatly affect the temperature reached by the sample and hence the ion intensities produced. ${ }^{10}$ This means that the time that the sample spends in the ion source has a critical impact on the results obtained. In addition, the measured temperature of the sample was found to reach only a fraction of the set temperature of the gas. These hitherto unreported phenomena have profound implications for quantitative experiments as we have found that ion intensities vary significantly with heating time. We have also shown that the components of binary mixtures desorb from the sample holder at different rates to when the same components are analysed individually, giving pseudosuppression effects. This leads to extremely time-dependent quantitative results.

As part of our research into the ionisation behavior of mixtures due to the thermal parameters of the DART source, we have developed a novel combined hot-stage microscopy-DART-MS system (HSM-DART-MS) which uses a miniaturised ceramic hot-stage in the ion source to give much more precise temperature control of samples than that obtained with the DART alone, as well as allowing visualisation of melting and desorption behavior through the integrated microscope.

Although a sample heating system for pyrolysis and thermal de-sorption is commercially available (IonRocket, BioChromato Inc., Japan), to the best of our knowledge, ours is the first example of a fully integrated instrument allowing controlled heating and cooling at variable rates while incorporating the additional benefit of optical imaging and color monitoring. The combined technique offers significant advantages over either hot-stage microscopy or DART-MS used alone.

We present experimental results from four diverse systems selected to exemplify the capability of the HSM-DART-MS system.

\section{EXPERIMENTAL SECTION}

Materials and supplies. Indium (ICTA Certified Reference Material 758) with a literature melting point of $156.6^{\circ} \mathrm{C}$ was used. ${ }^{11}$ Domestic Silicone bakeware was obtained from Kitchen Corner, Birmingham UK.

Alumina (99.95\%) was purchased from Alfa Aesar. Rhodamine B (97\%), 2-hydroxy1,4-naphthoquinone (97\%) and 2-hydroxybenzoic acid (99\%) were all purchased from Sigma Aldrich (Gillingham, UK). Anthracene-9,10-dione (certified reference material) was purchased from LGC Standards UK. Benzaldehyde (98+\%) was purchased from Acros Organics.

Most materials used in this work were not significantly hazardous, although the usual safety precautions were exercised. The technique involves volatilisation of chemicals in open air but the quantities used were small (a few $\mathrm{mg}$ ) and care was taken around the instrument during analyses. 
Table 1. Chemical structures and physical data for analytes used in the study

\begin{tabular}{|c|c|c|}
\hline Material / Structure & RMM g/mol & Melting point / \\
\hline Rhodamine B & 478.2 & 210 (decomp) \\
\hline 2-hydroxy-1,4-naphthoqu & 174.2 & $191-193$ \\
\hline Anthracene-9,10-dione & 208.2 & 283-287 \\
\hline 2-hydroxybenz & 138.1 & $158-160$ \\
\hline Benzaldehyde & 106.0 & -26 \\
\hline
\end{tabular}

HSM-DART-MS system. This instrumental configuration (Figure 1) requires a miniaturised hot-stage which has been constructed by us in-house, since commercially-available hot-stages are too large and have the wrong geometry to fit into the DART ion source. The new hot-stage consists of a ceramic block containing a Nichrome heating element and a low-mass thermocouple to measure the temperature of the block. The system is controlled by a PC utilising bespoke software extending on prototype code developed by the project team. The hot-stage is fully programmable and can operate both isothermally and with selectable temperature ramps to support both heating and cooling.

Images, RGB values (color) and mass spectra were recorded for every experiment; selected data are displayed within.

The configuration of the instrument means that material evolved from the sample during heating (via desorption, volatilisation or decomposition) is ionised by the flow of metastable helium atoms and drawn into the mass spectrometer due to its vacuum. This is subtly different from the operation of a conventional DART source where the sample itself is usually within the flow of heated helium. 


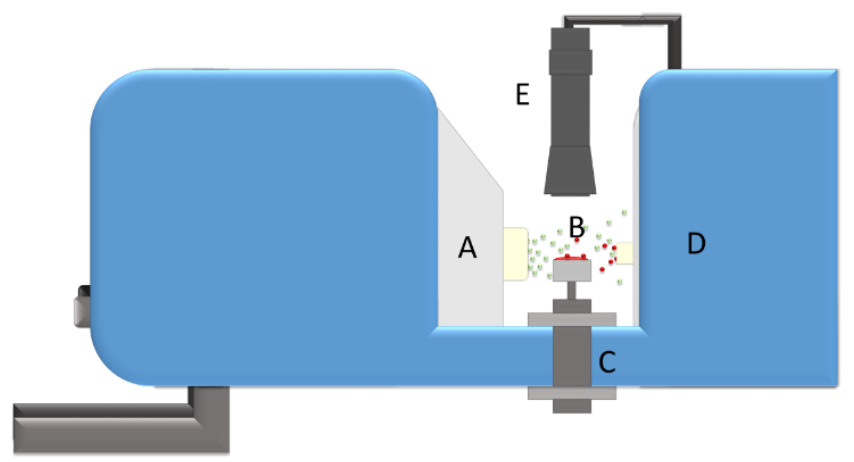

Figure 1. Hot-stage microscope-DART-MS ion source. A: DART outlet; B: sampling area; C: hot-stage; D: mass spectrometer inlet; E: digital microscope.

HSM-DART parameters. The DART was set up to provide a gas flow rate of 1.5 $\mathrm{Lmin}^{-1}$; the temperature was set to $30^{\circ} \mathrm{C}$. The voltages used were as follows: needle voltage $3 \mathrm{kV}$, discharge electrode $400 \mathrm{~V}$, grid electrode $400 \mathrm{~V}$. Samples were placed in $6 \mathrm{~mm}$ Inconel pans of the type typically used in many commercial thermal analysis instruments.

Images are continuously displayed and are saved as .jpeg files at definable time or temperature intervals, typically $5^{\circ} \mathrm{C}$.

HSM data: time, temperature and RGB values were saved as comma separated variables (.csv) files at one second intervals.

Mass spectrometer parameters. Samples were analysed on a Bruker Esquire HCT ion trap mass spectrometer. The mass range used was $100-2800 \mathrm{Th}$, in positive ion mode, using a maximum accumulation time of $200 \mathrm{~ms}$ and a Smart Target of 200,000 . The capillary voltage was set to $4 \mathrm{kV}$ and the source temperature set to $300{ }^{\circ} \mathrm{C}$.

Data processing. The data from the hot-stage microscope were plotted directly in Excel (Microsoft). Mass spectral data were ex-ported as .ascii files. An Excel macro was used to extract data corresponding to nominal masses of ions of interest using a window of $\pm 0.5 \mathrm{Th}$. The two data sets were combined in Excel using the VLOOKUP function. Mass spectral data were smoothed using moving window averaging of, typically, 5-10 data points (approximately $6 \mathrm{sec}$.). Color values are extracted in real time from a user-defined area within the optical field of view; the pixels within this area are summed to give $\Sigma R G B$ values. In addition, $\Delta R G B$ values, the change in overall color from the start of the experiment, can also be obtained. This gives a measure of the sample's color history and represents the degree of color change during analysis.

Calibration. External calibration of the temperature of the HSM was carried out using the method typically used for calibration of thermal analysis instruments, which involves monitoring the melting of temperature calibration standards, such as indium, tin or lead. Typically, a standard (5-100 mg) was heated at an appropriate heating rate for the experiments to be carried out and the melting monitored through $\triangle \mathrm{RGB}$ measurements. A typical temperature calibration utilised a graph plotting literature 
melting point against measured melting point for three standards. The linear regression equation was determined and used to correct subsequent measurements.

The mass spectrometer was calibrated according to the manufacturer's procedures.

Silicone study. A sample $(5 \times 5 \times 0.5 \mathrm{~mm})$ was cut from the silicone cookware and put into a DSC pan which was placed onto the hot-stage and subjected to a temperature program of $0.8^{\circ} \mathrm{Cmin}^{-1}$ to $200^{\circ} \mathrm{C}$, followed by cooling at the same rate to $50^{\circ} \mathrm{C}$.

Full scan mass spectra were acquired in positive ion mode and extracted ion chromatograms (EICs) of masses corresponding to previously published oligomer masses of PDMS were plotted. ${ }^{12}$

Thermal separation experiments. An approximately equal mass mixture of 2 hydroxy-1,4-naphthoquinone, anthracene-9,10-dione and 2-hydroxybenzoic acid was prepared by placing the compounds (approximately $2 \mathrm{mg}$ ) into the aluminium pan side by side.

Two experiments were performed: an initial linear heating at a rate of $5^{\circ} \mathrm{Cmin}^{-1}$ and a more complex program designed to assist resolution of the thermal processes alternating between $5{ }^{\circ} \mathrm{Cmin}^{-1}$ and $0.5^{\circ} \mathrm{Cmin}^{-1}$ stages (illustrated in the Results and Discussion).

Full scan mass spectra were acquired in positive ion mode and EICs of masses corresponding to protonated molecules of each compound were plotted (139 Th for 2-hydroxybenzoic acid, 175 Th for 2-hydroxy-1,4-naphthoquinone, 209 Th for anthracene-9,10-dione).

Reaction monitoring. Benzaldehyde $(30 \mu \mathrm{L})$ and aqueous ammonia $(35 \%, 150 \mu \mathrm{L})$ were placed into a pan and subjected to a temperature program of $10^{\circ} \mathrm{Cmin}^{-1}, 30$ to $350^{\circ} \mathrm{C}$. Full scan mass spectra were recorded in positive ion mode and EICs of ions of interest were extracted.

Desorption of rhodamine B from alumina. Solutions of rhodamine B (either 25 or 100 $\mathrm{mg}$ in methanol, $10 \mathrm{~mL}$ ) were prepared. An aliquot $(100 \mu \mathrm{L})$ of stock solution was deposited onto alumina $(100 \mathrm{mg})$; the resulting mixture was then sonicated for 15 minutes. The solution was allowed to evaporate overnight. A sample of solid (10 mg) was removed for analysis and placed within a DSC pan. The sample was then exposed to a temperature program of $5^{\circ} \mathrm{Cmin}^{-1}, 30-350^{\circ} \mathrm{C}$. Full scan mass spectra were recorded and EICs of the $[\mathrm{M}-\mathrm{Cl}]^{+}$ion of rhodamine $\mathrm{B}$ (443 Th) were extracted.

\section{RESULTS AND DISCUSSION}

Calibration. Figure 2 shows the $\triangle R G B$ profile for the melting of a sample of indium after temperature calibration. The onset of the step is caused by an increase in reflectivity as the sample melts and corresponds well with the literature melting point. Each point corresponds to a temperature interval of $0.05^{\circ} \mathrm{C}$. 


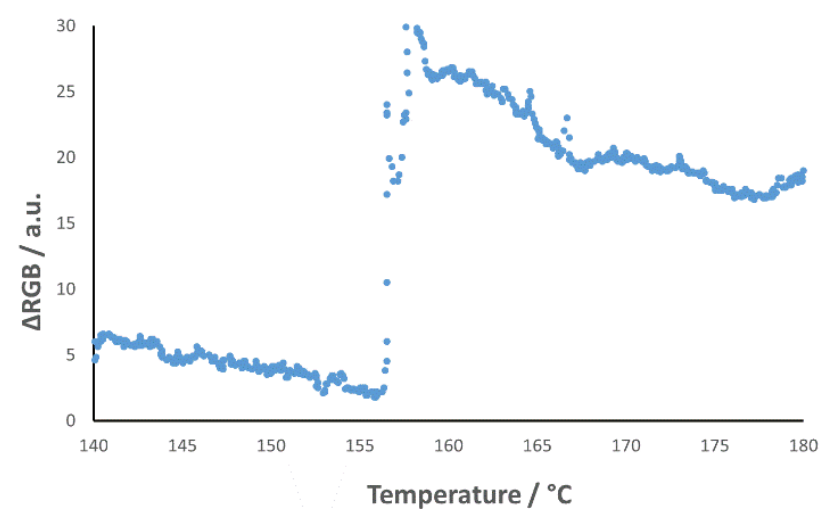

Figure 2. Plot of $\square$ RGB vs. temperature for the melting of a $66.3 \mathrm{mg}$ sample of indium at a heating rate of $5^{\circ} \mathrm{Cmin}^{-1}$.

Silicone study. This system was selected to assess the capability of the instrument to analyse high molecular weight materials based on the work of Gross ${ }^{12}$ who found that silicone household products such as baking tins evolved PDMS oligomers during heating.

Figure 3 shows the evolution of four PDMS oligomers from a sample of silicone cookware heated at $0.8 \mathrm{Cmin}^{-1}$ to $200{ }^{\circ} \mathrm{C}$ and then cooled at the same rate. The $\mathrm{m} / \mathrm{z}$ values of $684,758,832$ and 906 correspond to the ammoniated molecules for cyclic species comprised of $\left[\mathrm{Si}(\mathrm{Me})_{2} \mathrm{O}\right]_{n}$ units, where $\mathrm{n}=9-12$ (structures are given in Figure $\mathrm{S} 1$ in the Supporting Information together with a typical mass spectrum in Figure S2). For clarity, we have only presented data for four oligomers, although species ranging from $n=7$ to $n=25$ were observed over this temperature range; at higher temperatures oligomers up to $n=37$ were evolved.

It is interesting to note that the RMM of the oligomers evolved increases with increasing temperature. Although the ions at $\mathrm{m} / \mathrm{z} 684$ and 758 had achieved maximum evolution below $200{ }^{\circ} \mathrm{C}$, cooling the sample reduced the abundance of all species, as expected. At lower temperatures ions consistent with the presence of PEG, a known mold lubricant, were also observed. These results are consistent with previously published work ${ }^{12}$; there is ongoing work on this topic in our group.

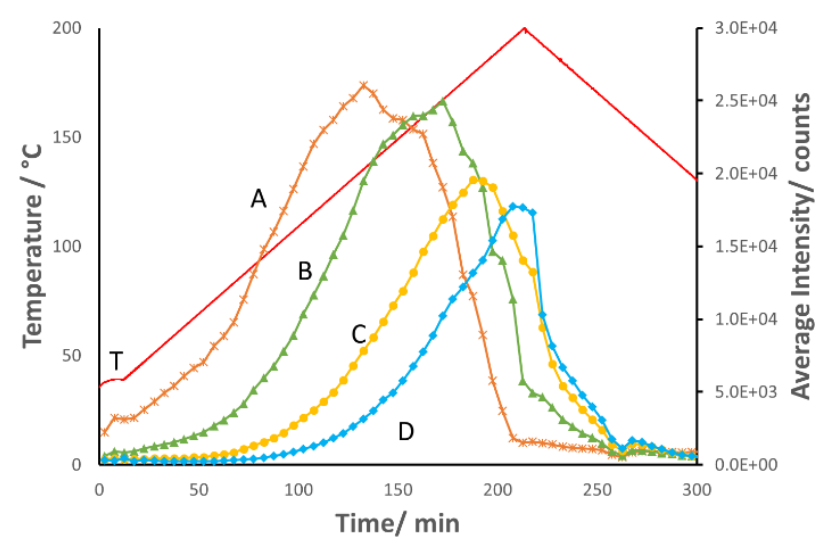

Figure 3. Extracted ion chromatograms showing the evolution of four PDMS oligomers as a function of temperature. Each point is an average of approximately 300 scans (equivalent to a five minute period). Key: A EIC of $\mathrm{m} / \mathrm{z} 684$; B EIC of $\mathrm{m} / \mathrm{z} 758$; C EIC of $\mathrm{m} / \mathrm{z}$ 832; D EIC of $\mathrm{m} / \mathrm{z} 906$; T temperature programme. 
The benefit of an integrated optical system is that it allows the morphology changes of a sample to be correlated with both color measurements and mass spectral data. This is demonstrated using silicone; in an experiment carried out over a wider temperature range, a sample of the silicone material was heated from ambient to $700{ }^{\circ} \mathrm{C}$ and optical images (Figure 4) and RGB values recorded. From the optical data, size measurements allowed the area of the sample to be calculated as a function of temperature (Figure 5). A gradual increase in sample area was observed up to $500^{\circ} \mathrm{C}$, correlating with a gradual change in color (Figure 5). Above $500^{\circ} \mathrm{C}$, when the silicone begins to decompose, a sharp decrease in sample area is noted, which is matched by a sharp change in the RGB profile as the sample decolorizes.

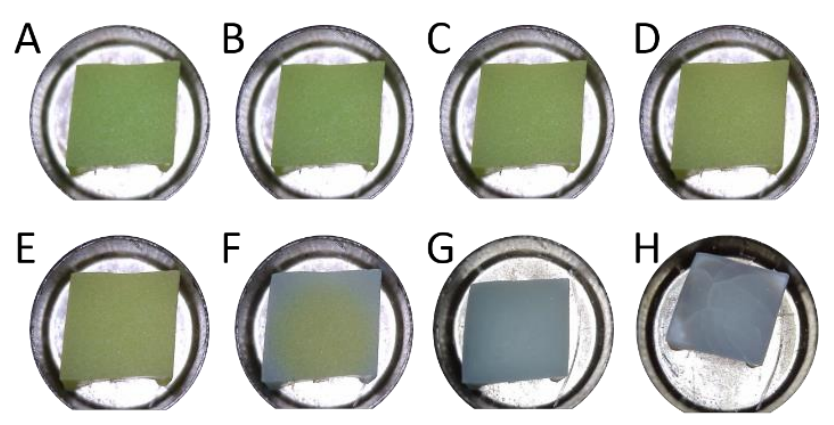

Figure 4. Microscope images of a sample of silicone cookware heated between $30^{\circ} \mathrm{C}$ and $700{ }^{\circ} \mathrm{C}$. Key: A $30{ }^{\circ} \mathrm{C}$; B $100^{\circ} \mathrm{C}$; C $200^{\circ} \mathrm{C}$; D $300^{\circ} \mathrm{C}$; E $400{ }^{\circ} \mathrm{C}$; F $500^{\circ} \mathrm{C}$; G 600 ${ }^{\circ} \mathrm{C} ; \mathrm{H} 700{ }^{\circ} \mathrm{C}$.

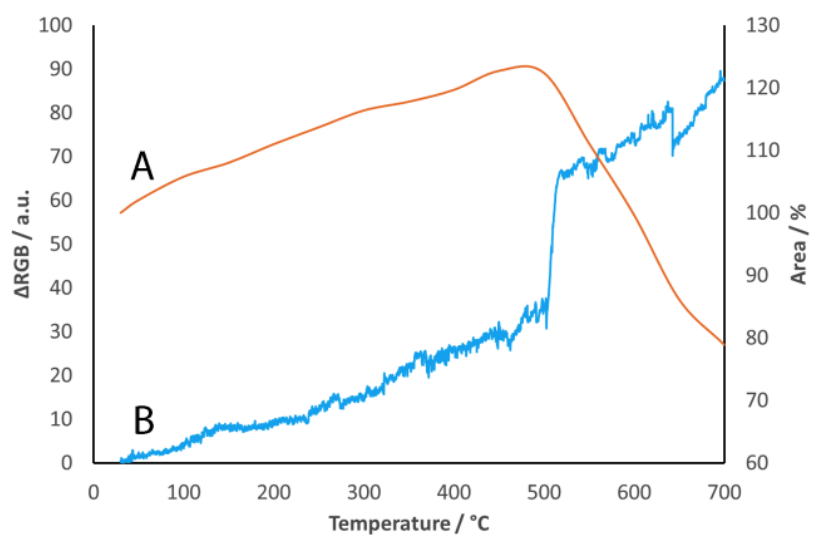

Figure 5. Optical data from a sample of silicone cookware heated from $30^{\circ} \mathrm{C}$ to 700 ${ }^{\circ} \mathrm{C}$ as a function of temperature. Key: A Relative change in area of the sample; $\mathrm{B}$ $\square$ RGB values.

Thermal separation experiments. A mixture of simple aromatic molecules with different melting points was analysed to determine the extent of thermal separation with differing heating programs. The first experiment used a basic, linear heating rate of $5{ }^{\circ} \mathrm{Cmin}^{-1}$. Based on the results from this, a second experiment was designed which utilised a more complex temperature profile, switching between fast and slow heating, to evaluate the impact on the resolution achieved. Figure 6 shows EICs of the protonated molecules of the three species. 


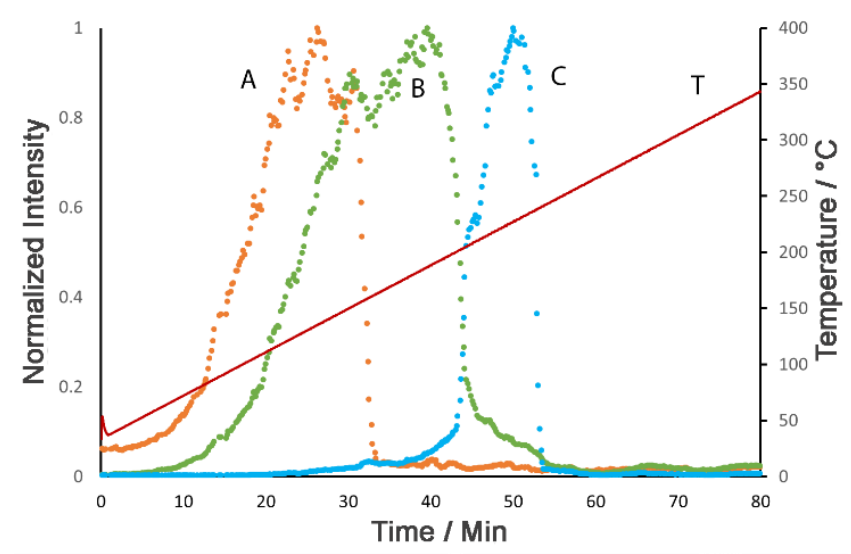

Figure 6. Extracted ion chromatograms showing thermal separation of the protonated adducts of three small molecules (2-hydroxy-1,4-naphthoquinone, 2-hydroxybenzoic acid and anthracene-9,10-dione) using a linear heating rate of $5{ }^{\circ} \mathrm{Cmin}^{-1}$ to $350{ }^{\circ} \mathrm{C}$. Each point is an average of 10 data points. Key: A EIC of $\mathrm{m} / \mathrm{z} 139$; B EIC of $\mathrm{m} / \mathrm{z} 175$; C EIC of $\mathrm{m} / \mathrm{z}$ 209; T temperature programme.

The results demonstrate that the evolution of each species from the mixture is observed in order of increasing melting point. The separation of 2-hydroxybenzoic acid and 2-hydroxy-1,4-naphthoquinone is less defined but anthracene-9,10-dione is wellseparated from the other compounds. The shape of the profiles arises from increased volatilisation after melting, until a maximum after which there is a decline primarily caused by removal of the material. The onset of the anthracene-9,10-dione signal matches the sharp decline in the 2-hydroxy-1,4-naphthoquinone signal; we do not believe this is the result of chemical reaction but rather complex pseudo-suppression effects, which have been observed previously in other mixtures using conventional DART analysis. ${ }^{10,13}$

It is generally recognised that slower heating rates give better thermal resolution, so a new method was developed using a combination of fast and slow heating rates (Figure 7). Here, the resolution of 2-hydroxybenzoic acid and 2-hydroxy-1,4naphthoquinone has been improved by this new method. Interestingly, some 2hydroxy-1,4-naphthoquinone is observed from the onset of melting of the 2hydroxybenzoic acid. This may be due to partial dissolution of the 2-hydroxy-1,4naphthoquinone in the liquid 2-hydroxybenzoic acid. Again, there is an indication that the onset of the anthracene-9,10-dione peak correlates to the abrupt termination of the 2-hydroxy-1,4-naphthoquinone signal. The drop in signal observed during the anthracene-9,10-dione peak has been observed in multiple experiments and appears to correlate to condensation and crystal formation at the edges of the pan, which is volatilised at higher temperatures (Figure S3 in SI). These results demonstrate how the ability of the instrument to implement more complex temperature profiles can separate components of mixtures, thereby simplifying interpretation of the resultant mass spectra. 


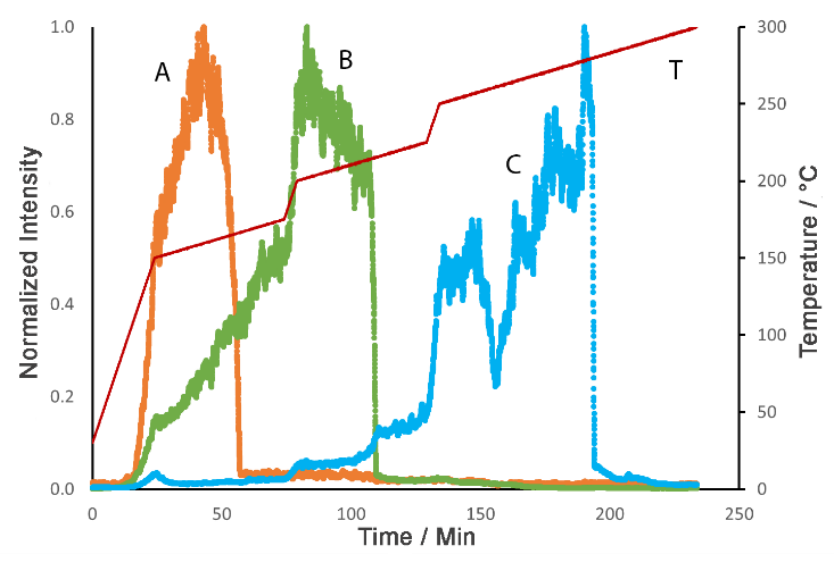

Figure 7. Extracted ion chromatograms showing thermal separation of the protonated adducts of three small molecules (2-hydroxy-1,4-naphthoquinone, 2-hydroxybenzoic acid and anthracene-9,10-dione) using a multi-stage temperature profile alternating between $5{ }^{\circ} \mathrm{Cmin}^{-1}$ and $0.5^{\circ} \mathrm{Cmin}^{-1}$ to a maximum of $300{ }^{\circ} \mathrm{C}$. Each point is an average of 6 data points. Key: A EIC of $\mathrm{m} / \mathrm{z}$ 139; B EIC of $\mathrm{m} / \mathrm{z} \mathrm{175}$; C EIC of $\mathrm{m} / \mathrm{z} 209$; T temperature programme.

Reaction monitoring. The potential of the instrument to monitor reaction processes in real time was demonstrated using the reaction of benzaldehyde with aqueous ammonia to form hydrobenzamide (Scheme S1 in SI).

Figure 8 shows that as the temperature starts to increase benzaldehyde is evolved until approximately $90^{\circ} \mathrm{C}$ when it starts to decline as the product is observed. The rate of evolution of hydrobenzamide increases until the starting material is exhausted and then declines as the remaining material is desorbed.

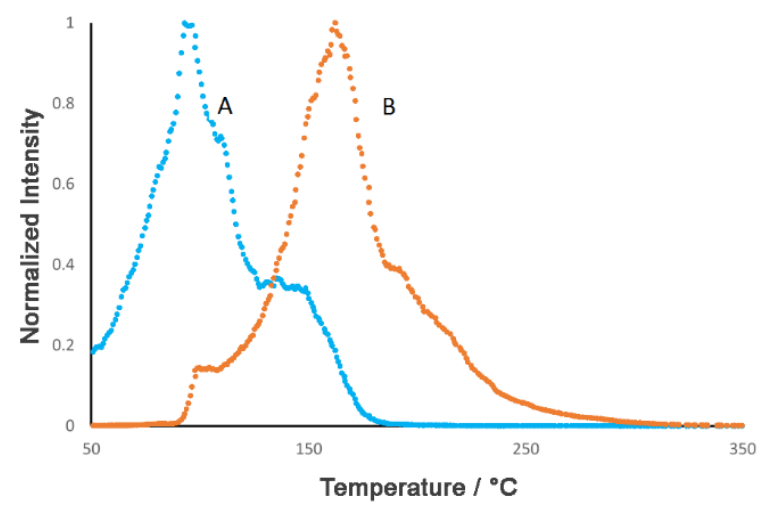

Figure 8. Extracted ion chromatograms showing reaction of benzaldehyde $(\mathrm{m} / \mathrm{z} 106)$ with aqueous ammonia to form hydrobenzamide $(\mathrm{m} / \mathrm{z} 299)$ during heating at $10^{\circ} \mathrm{Cmin}^{-}$ ${ }^{1}$ to $350^{\circ} \mathrm{C}$. Key: A EIC of $\mathrm{m} / \mathrm{z} 106$; B EIC of $\mathrm{m} / \mathrm{z} 299$.

We attribute the m/z 106 signal to the molecular ion of benzaldehyde ${ }^{14}[\mathrm{M}]^{+}$. and m/z 299 to the protonated form of hydrobenzamide $[\mathrm{M}+\mathrm{H}]^{+}$. The shoulder on the benzaldehyde peak is consistent with a proposed protonated reaction intermediate (Scheme 1) which has the same nominal mass as the starting material and was therefore extracted in our data processing procedure along with the benzaldehyde. 


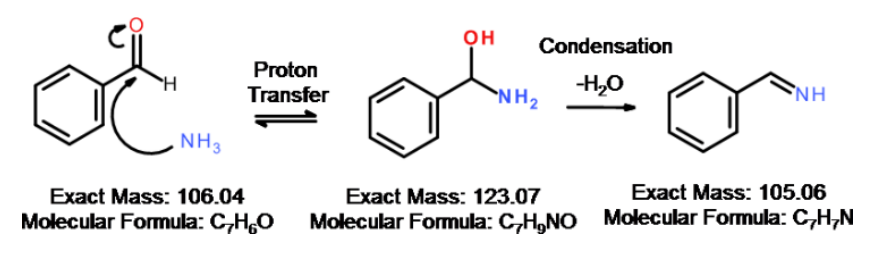

Scheme 1. Imine reaction intermediate formation through nucleophilic attack

Desorption of rhodamine $\mathbf{B}$ from alumina. This system was selected to demonstrate that it is possible to analyse samples adsorbed onto more complex matrices and to highlight the complementary information that the RGB values can provide. Two experiments were carried out using different amounts of rhodamine B applied to the alumina $(25 \mu \mathrm{g}$ and $100 \mu \mathrm{g})$. For both experiments it can be seen that the rhodamine $\mathrm{B}$ desorption starts at a temperature of approximately $170{ }^{\circ} \mathrm{C}$ and is complete by 270 ${ }^{\circ} \mathrm{C}$ (Figure 9).

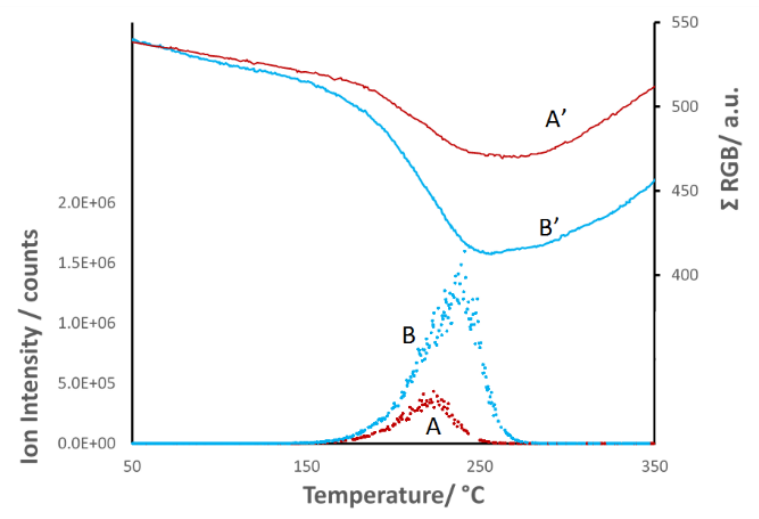

Figure 9. Extracted ion chromatograms and corresponding $\Sigma R G B$ traces showing desorption of two different amounts of rhodamine $B$ from alumina $\left(\mathrm{m} / \mathrm{z} 443[\mathrm{M}-\mathrm{Cl}]^{+}\right)$. The samples were heated at $5^{\circ} \mathrm{Cmin}^{-1}$ to $350{ }^{\circ} \mathrm{C}$. Key: A EIC of $\mathrm{m} / \mathrm{z} 443$ at $25 \mu \mathrm{g}$; B EIC of $\mathrm{m} / \mathrm{z} 443$ at $100 \mu \mathrm{g}$; A' $\square 443$ at $100 \mu \mathrm{grhodamine} B$ at $25 \mu \mathrm{g}$; B' $\square \mathrm{B}$ at $25 \mu \mathrm{g}$; Brhodamine B at $100 \mu \mathrm{g}$.

The signal intensity is reflective of the amount of sample present, integration of the EIC yields $3.77 \times 10^{6}$ and $14.7 \times 10^{6}$ counts.minutes for 25 and $100 \mu \mathrm{g}$ respectively, giving a ratio of 3.89 indicating that the technique may be at least semi-quantitative. The $\Sigma R G B$ profiles correlate well with the EICs. The initial decrease in the signal is due to desorption of the rhodamine $B$, causing a color change. The subsequent rise from $250{ }^{\circ} \mathrm{C}$ is caused by the darkening of the image as residual rhodamine $\mathrm{B}$ decomposes.

\section{CONCLUSIONS}

We have successfully developed a novel system which combines controlled heating, ambient ionisation mass spectrometry, microscopy and optical measurements. The real-time, multi-dimensional data that the instrument can provide offers extensive advantages over the individual techniques. The scope of the instrument has been demonstrated for a variety of systems including polymer analysis, reaction monitoring 
and desorption studies. Further investigations will assess its utility for quantitative measurements, including an assessment of its sensitivity and reproducibility. In addition, we intend to investigate the detection of analytes in more complex matrices. We also plan to modify the system to increase thermal resolution by incorporating feedback between the ion intensity or optical change and heating rate.

\section{ASSOCIATED CONTENT}

Supporting Information

A file of Supporting Information is provided which contains chem-ical structures of PDMS oligomers, a representative mass spectrum of the silicone sample, microscope images of the small molecule mixture, and a reaction scheme showing the conversion of benzal-dehyde to hydrobenzamide (PDF file).

\section{AUTHOR INFORMATION}

Corresponding author

Dr Lindsay P. Harding, Dept of Chemical Sciences, Univer-sity of Huddersfield, Queensgate, Huddersfield, HD1 3DH, UK. Phone (+44) 1484 472434. Email: I.p.harding@hud.ac.uk. Orcid ID: 0000-0003-3985-1251

Notes

The authors declare no competing financial interest.

Author Contributions

The manuscript was written through contributions of all authors. All authors contributed equally to the work. All authors have given approval to the final version of the manuscript.

\section{ACKNOWLEDGEMENTS}

The authors would like to thank Dr Gary Midgley, University of Huddersfield, for helpful discussions and the Strategic Research Infrastructure Fund and the University of Huddersfield for funding.

\section{REFERENCES}

1. Charsley, E.L. and Kamp, A.C.F., in H.G. Wiedemann (ed.) Thermal Analysis, Vol. 1, Birkhauser, 1972, 499

2. Vitez, I.M., Newman, A.W., Davidovich, M. and Kiesnowski, C., Thermochimica Acta, 1998, 342, 187-196

3. Haines, P.J., Thermochimica Acta, 1999, 340-341, 285-292

4. Matzakos, A.N. and Zygourakis, K., Rev. Sci. Instrum., 1993, 64(6), 1541-1548

5. Cody, R.B., Laramee, J.A. and Durst, H.D., Anal. Chem., 2005, 77(8), 2297-2302; Cody,

R.B., Laramee, J.A., Nilles, J.M. and Durst, H.D., JEOL News, 2005, 40(1), 8-12 
6. Jones, R.W., Cody, R.B. and McClelland, J.F., J. Forensic Sci., 2006, 51(4), 915-918; Lesiak, A.D.and Shepard, J.R.E., Bioanalysis, 2014, 6(6), 819-842.

7. Nei, D., Nakamura, N., Ishihara, K. and Kimura, M., Food Control, 2017, 75, 181-186

8. Antal, B., Kuki, A., Nagy, L. and Zsuga, M., Anal. Bioanal. Chem., 2016, 408, 5189-5198

9. Gross, J.H., Anal. Bioanal. Chem., 2014, 406, 63-80

10. Harding, L.P., Parkes, G.M.B. and Townend, J.D., Analyst, 2014, 139, 4176-4180

11. Thomas, H.P., Metrologia, 1990, 27, 3-10

12. Gross, J.H., J. Am. Soc. Mass Spectrom., 2015, 26(3), 511-521

13. Shelley, J.T. and Heftje, G.M., J. Anal. At. Spectrom., 2010, 25, 345-350

14. Hunter, E.P. and Lias, S.G., J. Phys. Chem. Ref. Data, 1998, 27(3), 413-656 